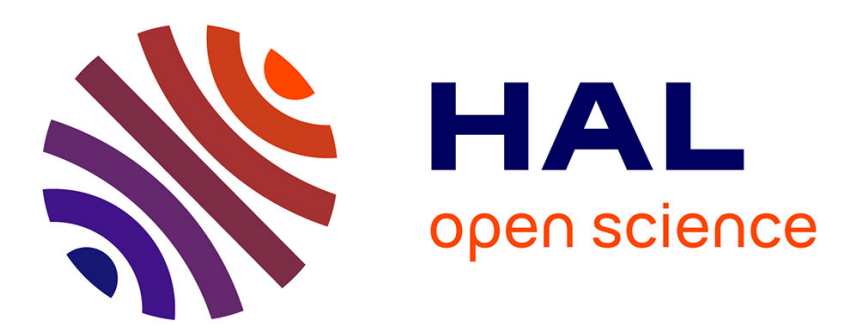

\title{
Mitochondrial energetic metabolism: A simplified model of TCA cycle with ATP production
}

Christine Nazaret, Margit Heiske, Kevin Thurley, Jean-Pierre Mazat

\section{To cite this version:}

Christine Nazaret, Margit Heiske, Kevin Thurley, Jean-Pierre Mazat. Mitochondrial energetic metabolism: A simplified model of TCA cycle with ATP production. Journal of Theoretical Biology, 2009, 258 (3), pp.455. 10.1016/j.jtbi.2008.09.037 . hal-00554511

\section{HAL Id: hal-00554511 \\ https://hal.science/hal-00554511}

Submitted on 11 Jan 2011

HAL is a multi-disciplinary open access archive for the deposit and dissemination of scientific research documents, whether they are published or not. The documents may come from teaching and research institutions in France or abroad, or from public or private research centers.
L'archive ouverte pluridisciplinaire HAL, est destinée au dépôt et à la diffusion de documents scientifiques de niveau recherche, publiés ou non, émanant des établissements d'enseignement et de recherche français ou étrangers, des laboratoires publics ou privés. 


\section{Author's Accepted Manuscript}

Mitochondrial energetic metabolism: A simplified model of TCA cycle with ATP production

Christine Nazaret, Margit Heiske, Kevin Thurley, Jean-Pierre Mazat

PII: S0022-5193(08)00493-1

DOI: doi:10.1016/j.jtbi.2008.09.037

Reference: YJTBI5299

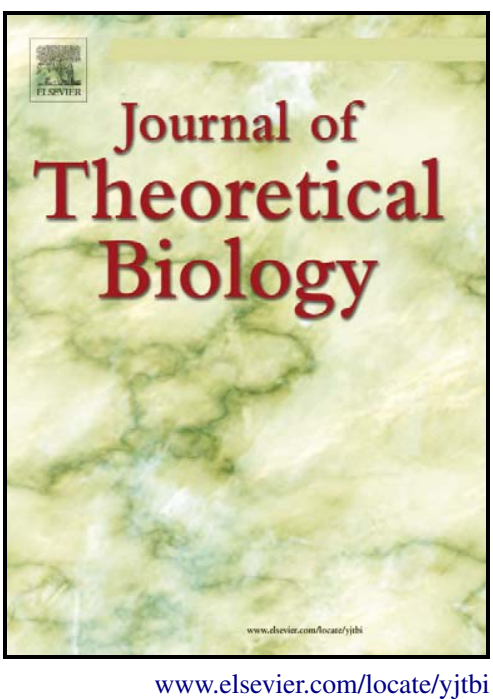

To appear in: $\quad$ Journal of Theoretical Biology

Received date: $\quad 12$ February 2008

Revised date: $\quad 18$ July 2008

Accepted date: 18 September 2008

Cite this article as: Christine Nazaret, Margit Heiske, Kevin Thurley and Jean-Pierre Mazat, Mitochondrial energetic metabolism: A simplified model of TCA cycle with ATP production, Journal of Theoretical Biology (2008), doi:10.1016/j.jtbi.2008.09.037

This is a PDF file of an unedited manuscript that has been accepted for publication. As a service to our customers we are providing this early version of the manuscript. The manuscript will undergo copyediting, typesetting, and review of the resulting galley proof before it is published in its final citable form. Please note that during the production process errors may be discovered which could affect the content, and all legal disclaimers that apply to the journal pertain. 


\title{
Mitochondrial energetic metabolism : A simplified model of TCA cycle with ATP production.
}

\author{
Christine Nazaret ${ }^{1}$, Margit Heiske ${ }^{3}$, Kevin Thurley ${ }^{3}$, Jean-Pierre Mazat ${ }^{2}$. \\ ${ }^{1}$ IMB UMR 5251-ESTBB and ${ }^{2}$ Inserm U688, Université de Bordeaux 2, 146 rue Léo-Saignat, F 33076, \\ Bordeaux-cedex, France. ${ }^{3}$ Humboldt Universität, Berlin, Germany.
}

Keywords : Mitochondria; energetic metabolism; respiratory chain; oxidative phosphorylation; ATP synthase; TCA cycle; dynamical system; existence of steady state;

Abstract

Mitochondria play a central role in cellular energetic metabolism. The essential parts of this metabolism are the Tricarboxyl-acid (TCA) cycle, the respiratory chain and the Adenosine Tri-Phosphate (ATP) synthesis machinery. Here a simplified model of these three metabolic components with a limited set of differential equations is presented. The existence of a steady state is demonstrated and results of numerical simulations are presented. The relevance of a simple model to represent actual in vivo behavior is discussed.

\section{Introduction}

Mitochondria are small organelles found in most living cells. They are sometimes described as cellular power plants because one of their functions is to convert organic materials into energy in the form of ATP. A mitochondrion is composed of an inner space called matrix containing a highly concentrated mixture of enzymes and chemical species. The matrix is enclosed by two membranes with very different properties. The inner membrane is highly impermeable to ions and molecules which require special membrane transporters to enter and exit the matrix, whereas the outer membrane is permeable to many molecules. In this work, only the matrix and the inner mitochondrial membrane will be considered. The mitochondrial energy metabolism involves three main sets of biochemical reactions : the tricarboxylic acid cycle (TCA cycle or Krebs cycle), the respiratory chain and the ATP synthesis machinery (see fig. 1). The mitochondrial energetic metabolism has extensively been modeled since the pioneering works of Garfinkel $[17,18]$, Wilson [50] and Bohnensack [12]. Korzeniewski [27] developed a more complex model also based on ordinary differential equations, which was applied to isolated mitochondria or to intact tissues (muscle, heart and liver). Cortassa [14] developed a model including the TCA cycle, the transports of $\mathrm{Ca}^{2+}$ across the mitochondrial inner membrane and the regulation of dehydrogenases by $\mathrm{Ca}^{2+}$. The interest of this model is to match the supply of mitochondrial energy to the cellular demand, through the variations of intracellular and intramitochondrial $\mathrm{Ca}^{2+}$. More recently, Beard [10] and $\mathrm{Wu}$ [53] proposed "A biophysical model of the mitochondrial respiratory system and oxidative phosphorylation" mainly applied to cardiac mitochondria.

But as the set of equations of the models is usually very large, mathematical analysis of such models is quite hard and rarely performed. Our aim is to develop a simple model of the mitochondrial energetic metabolism based on a limited set of differential equations. It involves a simple phenomenological representation of the respiratory chain and of ATP synthesis machinery by only one phenomenological equation in each case and a reduced model of TCA cycle by lumping consecutive reactions while keeping the possible design of this part of the metabolism in two sub-cycles.

With this very simple and reduced model, our goal is to simulate the most important properties of the 3-part mitochondrial energy metabolism i.e. $i$ ) the links between the 3 parts: ATP synthesis, respiratory chain and TCA cycle and ii) the coordinate response when ATP demand is varied: responses of the transmembrane potential, of the rate of respiration and of the flux through the TCA cycle. In addition, we will impose iii) keeping in a physiological range the concentrations of the few intermediate metabolites considered in TCA cycle. We will give mathematical properties of the model (existence of a unique non-negative solution globally defined on $[0,+\infty)$, existence of stationary state). Then, we will perform some simulations to illustrate the interest of using a simple model to emphasize actual in vivo behaviors.

\section{The model}

The essential parts of the mitochondrial energetic metabolism responsible for ATP production are summarized in fig. 1A. They involve the TCA cycle, the respiratory chain $(R C)$ which generates a proton gradient, the ATP synthase (Asase), the adenine-nucleotide-translocator (ANT) and a proton leak through the membrane (L). We will describe in the following how we model these different parts in a simplified way. 


\subsection{Inside the matrix : TCA cycle modelling}
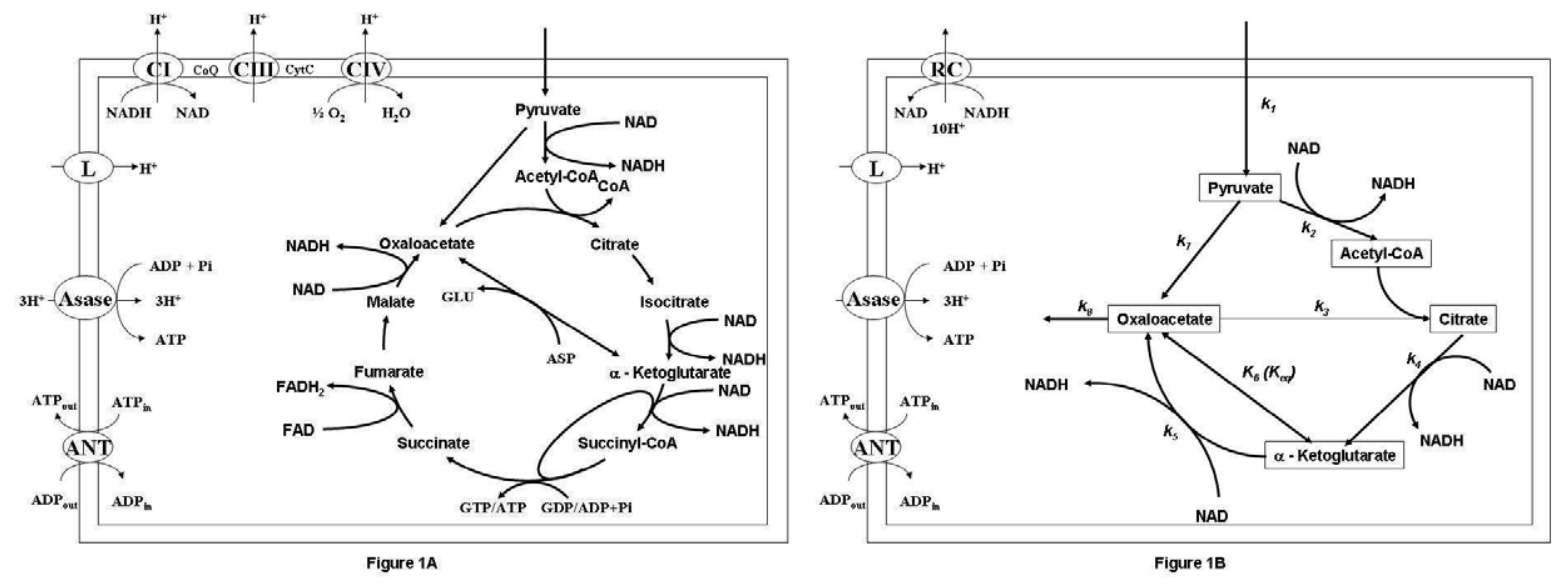

Figure 1: Essential parts of mitochondrial energetic metabolism (A) and our model (B)

The TCA cycle is a central part of the energetic metabolism that contributes to the generation of ATP by the chemical breakdown of carbohydrates, fats and proteins. It is a series of chemical reactions catalysed by enzymes occurring in the inside of mitochondria, called the matrix, and which can be disposed circularly (see fig. 1A). It has been recognized since a long time that the TCA cycle can be split into two mini cycles by transaminases linking directly $\alpha$-ketoglutarate to oxaloacetate $([41,26,35])$. In our model the two mini-cycles are assembled each in only one reaction (see fig. 1B): one reaction representing aconitase plus isocitrate dehydrogenase (reaction 4 where reaction $i$ corresponds to reaction $\stackrel{k_{i}}{\longrightarrow}$ of fig. 1B), the other assembling $\alpha$-ketoglutarate dehydrogenase, succinyl-CoA synthase, fumarase and malate dehydrogenase (reaction 5). Both reactions are thought to be irreversible because in each case, at least one of the elementary reactions is irreversible : reaction 4 because isocitrate dehydrogenase is irreversible and also reaction 5 because succinate dehydrogenase, which is part of Complex II of the respiratory chain, is irreversible. One of the main features of the TCA cycle is the reduction of NAD to NADH. The reduction of one NAD can be attributed to the first assembling reaction 4 and two NAD are reduced by reaction 5. In addition another NAD reduction is linked to pyruvate dehydrogenase (reaction 2) following the entry of pyruvate (reaction 1) and is also irreversible ([48]). Another quasi-irreversible fate of pyruvate goes through the pyruvate carboxylase (reaction 7); the quasi-irreversibility in this case is due to the very small concentration of oxaloacetate. The modelling of the rate equations by taking into account the "quasi-stationary state hypothesis" leads to quite sophisticated formulas (see [44] for more details). For the sake of simplicity we use the mass action law as indicated below.

$$
\begin{array}{lll}
\text { if irreversible } & A+B \longrightarrow C+D & \frac{d C}{d t}=k A B \\
\text { otherwise reversible } & A+B \longleftrightarrow C+D & \frac{d C}{d t}=k\left(A B-\frac{C D}{K_{e q}}\right)
\end{array}
$$

with $k$ the rate of the forward reaction and $K_{e q}$ the equilibrium constant of the reaction approximated by $K_{e q}=\frac{C_{e q} D_{e q}}{A_{e q} B_{e q}}$ where $X_{e q}$ are the concentration of the species $X$ at equilibrium (when the forward reaction rate equals the backward reaction rate).

In order that the system reaches a steady state, it is necessary to add some outputs to the TCA cycle through oxaloacetate (reaction 8) and through NADH with a simple reaction representing the respiratory chain $(\mathrm{RC})$ establishing a transmembrane potential $\Delta \psi$ modulated by a variable leak (L) and ATP synthesis (Asase). The effect of taking reactions 3 and 7 reversible has also been numerically studied.

\subsection{At the membrane: The respiratory chain, the ATP-synthase, the adenine-nucleotide- translocator and the proton leak}

- Transmembrane potential

The inner mitochondrial membrane is a selective barrier necessary for the coupling of the respiratory chain with the ATP synthesis. All of these mechanisms lead to changes in the proton 
concentration inside the mitochondrial matrix, which is generally lower than outside. This creates a difference of potential $\Delta \psi$ through this membrane. We will consider this as a capacitor (as in [32]):

$$
\Delta \psi=\frac{Q}{C}
$$

where $Q$ is the charge, and $C$ the capacitance. Empirical values can be found in the literature for $C$ (see table 2) that directly converts the concentration of protons into an electrical potential $\Delta \psi=\frac{H_{e}^{+}(t)-H^{+}(t)}{C}$ where the index $e$ stands for outside of membrane. Thus, if we hypothesize that $H_{e}^{+}$concentration is constant due to the large buffer volume outside the mitochondrial matrix, we obtain :

$$
\Delta \psi^{\prime}(t)=-\frac{1}{C} H^{+^{\prime}}(t)
$$

- Respiration

The respiratory chain is a very complex mechanism (see transmembrane complexes I to IV on fig. 1) which is globally irreversible. We assemble all the respiratory chain complexes depicted in fig. $1 \mathrm{~A}$ in one entity (RC) in fig. $1 \mathrm{~B}$ with a rate function $v_{\text {resp }}$. The oxidation of one molecule of NADH provides the energy to transport 10 protons outside the matrix into the intermembrane space. This can be simplified by two coupled reactions

$$
\left\{\begin{array}{ccc}
\mathrm{NADH}+\frac{1}{2} \mathrm{O}_{2}+\mathrm{H}^{+} & \longrightarrow & \mathrm{NAD}^{+}+\mathrm{H}_{2} \mathrm{O} \\
10 \mathrm{H}^{+} & \stackrel{v_{\text {resp }}}{\longrightarrow} & 10 \mathrm{H}_{e}^{+}
\end{array}\right.
$$

The rate of this reaction $v_{\text {resp }}$ depends on the $N A D H$ concentration and on the protons $H^{+}$. Since the protons are charged, the dependence on $\mathrm{H}^{+}$is not only due to the difference of concentration in $H^{+}$expressed as a $\Delta p H$ (osmotic effect) but also to an electrical effect expressed as a $\Delta \psi$ (see above). Both effects are summarized in the electrochemical potential difference in protons by the formula

$$
\Delta \tilde{\mu}=\Delta \mu+F \Delta \psi
$$

where $\Delta \mu=2.3 R T\left(p H-p H_{e}\right)=2.3 R T \log \left(\frac{H_{e}^{+}}{H^{+}}\right)$is the chemical part and $F \Delta \psi$ the electrical one with $F$ the Faraday constant, $R$ the gas constant and $T$ the temperature in Kelvin. For liver mitochondria a $\Delta p H=p H_{i}-p H_{e}$ of 0.75 and a $\Delta \psi$ of $168 \mathrm{mV}$ have been measured ([14]). At $T=$ $298^{\circ} \mathrm{K}$, we found $\Delta \mu \approx 4300 \mathrm{~J} / \mathrm{mol}$ and $F \Delta \psi \approx 16200 \mathrm{~J} / \mathrm{mol}$ which is approximately four times higher than $\Delta \mu$. Thus to simplify our modelling, the chemical effect $\Delta \mu$ is taken to be equal to $20 \%$ of $F \Delta \psi$, and thus the respiration rate will only depend on $\Delta \psi$ and of course, as mentioned above, on the NADH (or NAD) concentration. The rate function $v_{\text {resp }}$ has two essential experimental properties: a michaelis (hyperbolic) dependency of the $N A D H$ concentration with a saturating effect and a complete inhibition at high $\Delta \psi$ (the $H^{+}$extrusion by the respiratory chain built the $\Delta \psi$ which acts against the extrusion of $\mathrm{H}^{+}$).

To calculate these dependencies (see for instance [32, 12]), we chose a differentiable function which describes these phenomenological proprieties of the respiration rate: a saturation curve for $N A D H$, a nearly constant rate at $\Delta \psi$ of up to $\Delta \psi_{m}=150 \mathrm{mV}$, then a decrease towards 0 when $\Delta \psi$ increases (see fig. 2)

$$
v_{\text {resp }}(N A D, \Delta \psi)=k_{\text {resp }} \frac{N_{t}-N A D}{K+N_{t}-N A D} \frac{1}{1+\exp \left(a\left(\Delta \psi-\Delta \psi_{m}\right)\right)}
$$

where $N_{t}$ is the total nicotinamide adenine dinucleotide concentration $N_{t}=N A D+N A D H$ which is constant.

- ATP synthesis

To produce ATP (the natural direction for the reaction is the hydrolysis of ATP to produce ADP $+P i$ instead of the ATP synthesis), the ATP synthase needs the energy supplied by the entry of 3 protons into the matrix space. This can be expressed by the two coupled reactions

$$
\left\{\begin{array}{ccc}
A D P+P i & \longleftrightarrow & A T P+H_{2} \mathrm{O} \\
3 H_{e}^{+} & \stackrel{v_{A T P}}{\longleftrightarrow} & 3 H^{+}
\end{array}\right.
$$




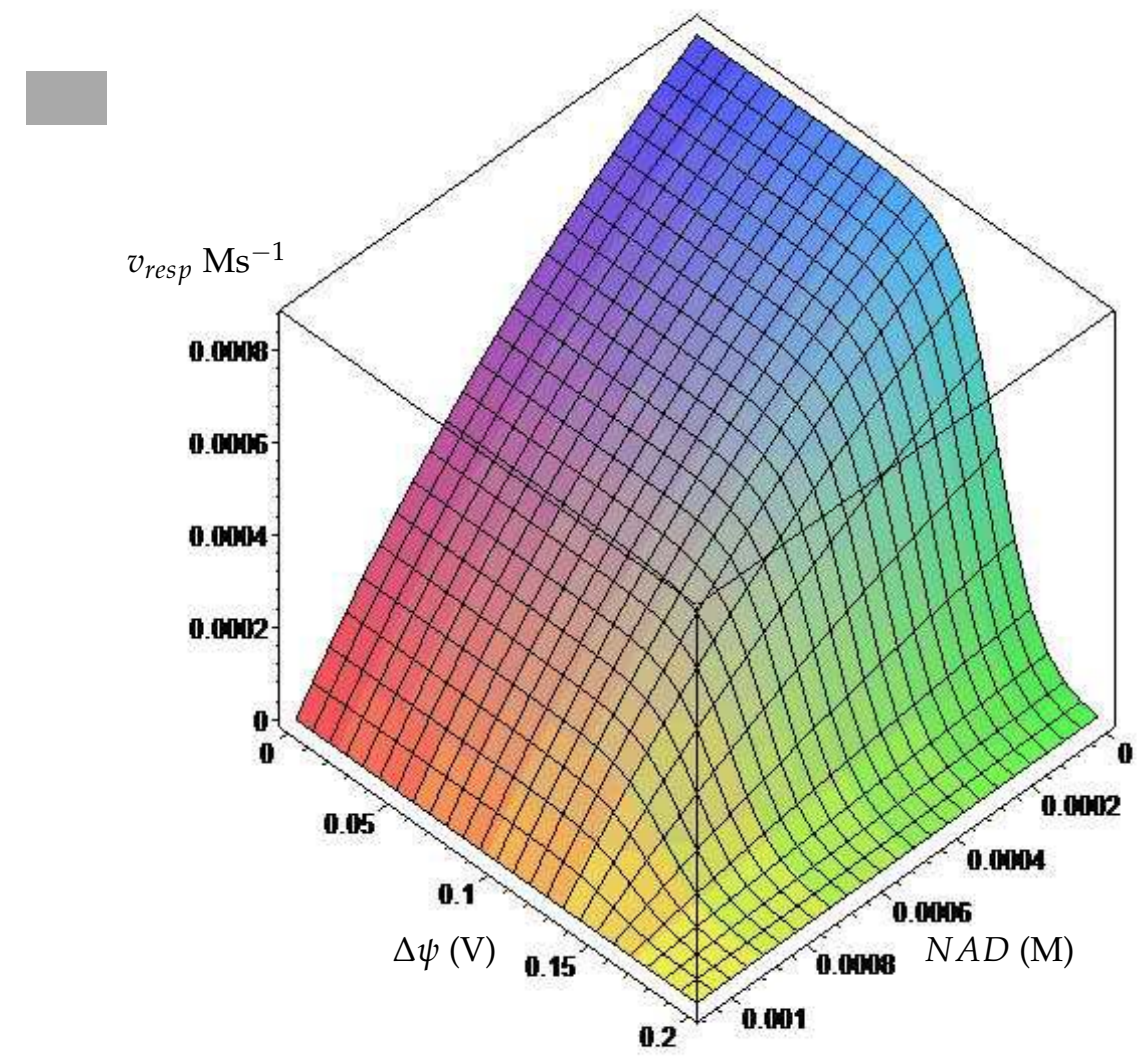

Figure 2: Respiration rate modelling $v_{\text {resp }}$

$$
k_{\text {resp }}=2.5 \mathrm{mMs}^{-1}, K=0.002 \mathrm{M}, N_{t}=1.07 \mathrm{mM}, a=100 \mathrm{~V}^{-1}, \Delta \psi_{m}=0.15 \mathrm{~V}
$$

An apparent equilibrium constant can be defined for the first reaction : $K_{a p p}=\frac{A T P_{e q}}{A D P_{e q} P i_{e q}}$ (the convention is that $\mathrm{H}_{2} \mathrm{O}$ is omitted in the equilibrium expression for $K_{\text {app }}$ when reactions in dilute solutions are considered). We also define $\Gamma=\frac{A T P}{A D P P i}=\frac{A T P}{\left(A_{t}-A T P\right) P i}$ where $A_{t}$ is the total adenine nucleotide concentration $A_{t}=A T P+A D P$ which is constant. The free Gibbs energy variation of this reaction can be written as follows

$$
\Delta G_{1}=-R T \ln \left(\frac{K_{a p p}}{\Gamma}\right) .
$$

The equilibrium constant is much more in favor of $A D P\left(K_{\text {app }} \approx 10^{-6}\right)$. But mitochondria are able to maintain a ratio $\Gamma$ which is far away from equilibrium to synthesize ATP: it requires an input of energy given by the second reaction $3 H_{e}^{+} \longleftrightarrow 3 H^{+}$.

The overall free-energy change $\Delta G$ of the coupled reactions is equal to the sum of the free-energy changes of both: $\Delta G=\Delta G_{1}+\Delta G_{2}$ where $\Delta G_{2}$ is the free Gibbs energy of the second reaction, i.e. the energy released by the protons crossing the inner membrane. Because we hypothesized that the chemical potential variation of the proton is equal to $20 \%$ of $F \Delta \psi$, the free energy for one mole of protons to cross the inner membrane is given by

$$
\Delta G_{\text {transport }}=1.2 \mathrm{~F} \Delta \psi .
$$

Thus, the free Gibbs energy $\Delta G$ of the coupled reactions is given by (see fig. 3)

$$
\Delta G(A T P, \Delta \psi)=-R T \ln \left(\frac{K_{a p p}}{\Gamma}\right)-3 \Delta G_{\text {transport }} .
$$

The direction of the reaction is given by the sign of $\Delta G$. If $\Delta G<0$, the phosphorylation of ADP in order to obtain ATP will proceed and the difference of potential $\Delta \psi$ will decrease. Otherwise the direction of the reaction will be in favor of ATP hydrolysis with proton extrusion and a building of the $\Delta \psi$ by the ATP synthase.

From $\Delta G=0$, we evaluate a critical threshold for ATP depending on the potential $\Delta \psi$, denoted 


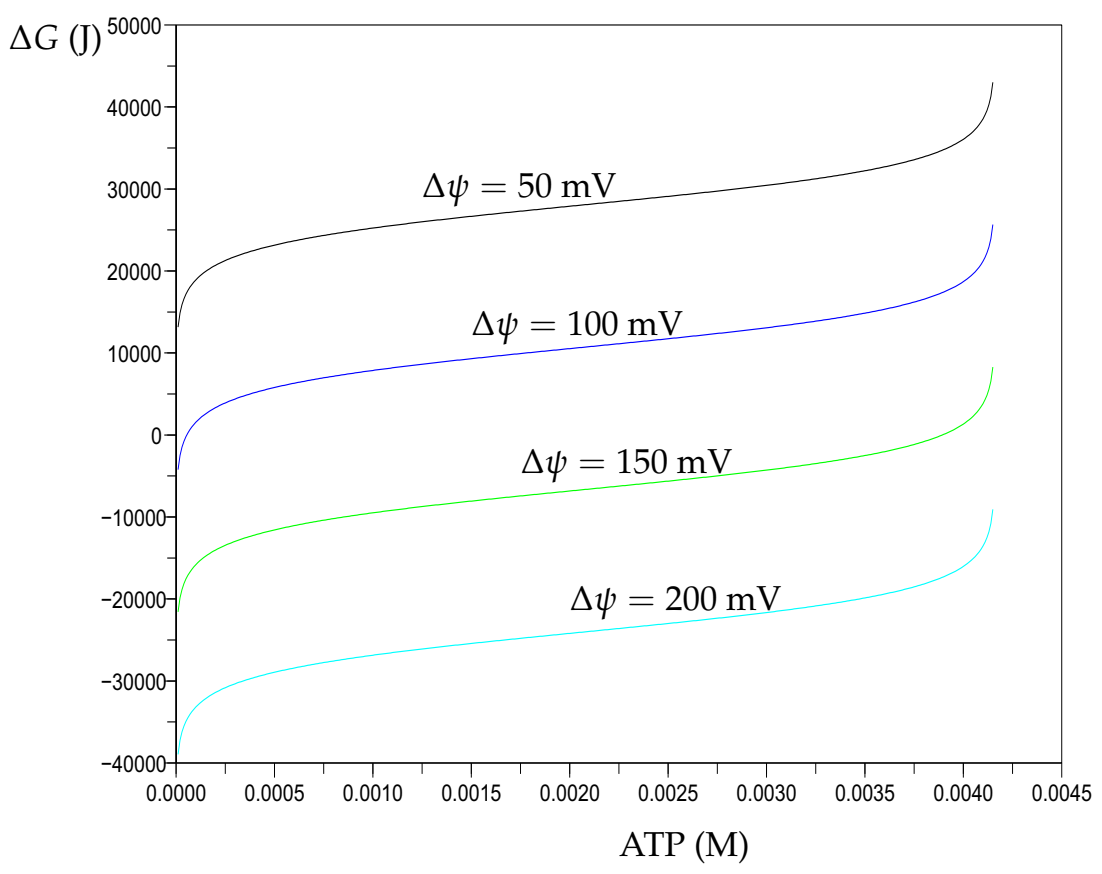

Figure 3: Free Gibbs energy $\Delta G$ of ATP synthase as a function of ATP concentration at different $\Delta \psi$ values indicated on the curves. $R=8.314 \mathrm{~J}(\mathrm{~mol} \mathrm{~K})^{-1}, T=298 \mathrm{~K}, F=96485 \mathrm{C} \mathrm{mol}^{-1}, K_{a p p}=4.4 \mu \mathrm{M}$.

$A T P_{\text {crit }}$ (see fig. 4)

$$
A T P_{\text {crit }}(\Delta \psi)=\frac{A_{t}}{1+\frac{\exp \left(\frac{-3 \Delta G_{\text {transport }}}{R T}\right)}{K_{\text {app }} P i}}
$$

and we set for the rate of reaction (see fig. 5)

$$
v_{A T P}(A T P, \Delta \psi)=k_{A T P}\left(\frac{2}{1+\exp \left(b\left(A T P-A T P_{c r i t}(\Delta \psi)\right)\right)}-1\right) .
$$

If $A T P<A T P_{\text {crit }}$ then $v_{A T P}>0$ and the phosphorylation of $A D P$ will proceed. Otherwise the direction of the reaction will be in favor of ATP hydrolysis.

- The Adenine Nucleotide Translocator is a mitochondrial protein that allows the exchange of ATP and $A D P$ molecules across the inner mitochondrial membrane: $A T P+A D P_{e} \stackrel{v_{A N T}}{\longrightarrow} A D P+A T P_{e}$. This mechanism can be simplified by

$$
A T P \stackrel{v_{A N T}}{\longrightarrow} A D P
$$

Since $A T P$ and $A D P$ exhibit a difference of charge $\left(A T P^{4-}\right.$ and $\left.A D P^{3-}\right)$, the exchange of $A D P$ against $A T P$ is accompanied by a concomitant decrease of $\Delta \psi$. For this reason, $v_{A N T}$ also appears in the equation expressing the variation of $\Delta \psi$. We assume that the adenine-nucleotidetranslocator process follows a simple law of mass action:

$$
v_{A N T}=k_{A N T} A T P .
$$




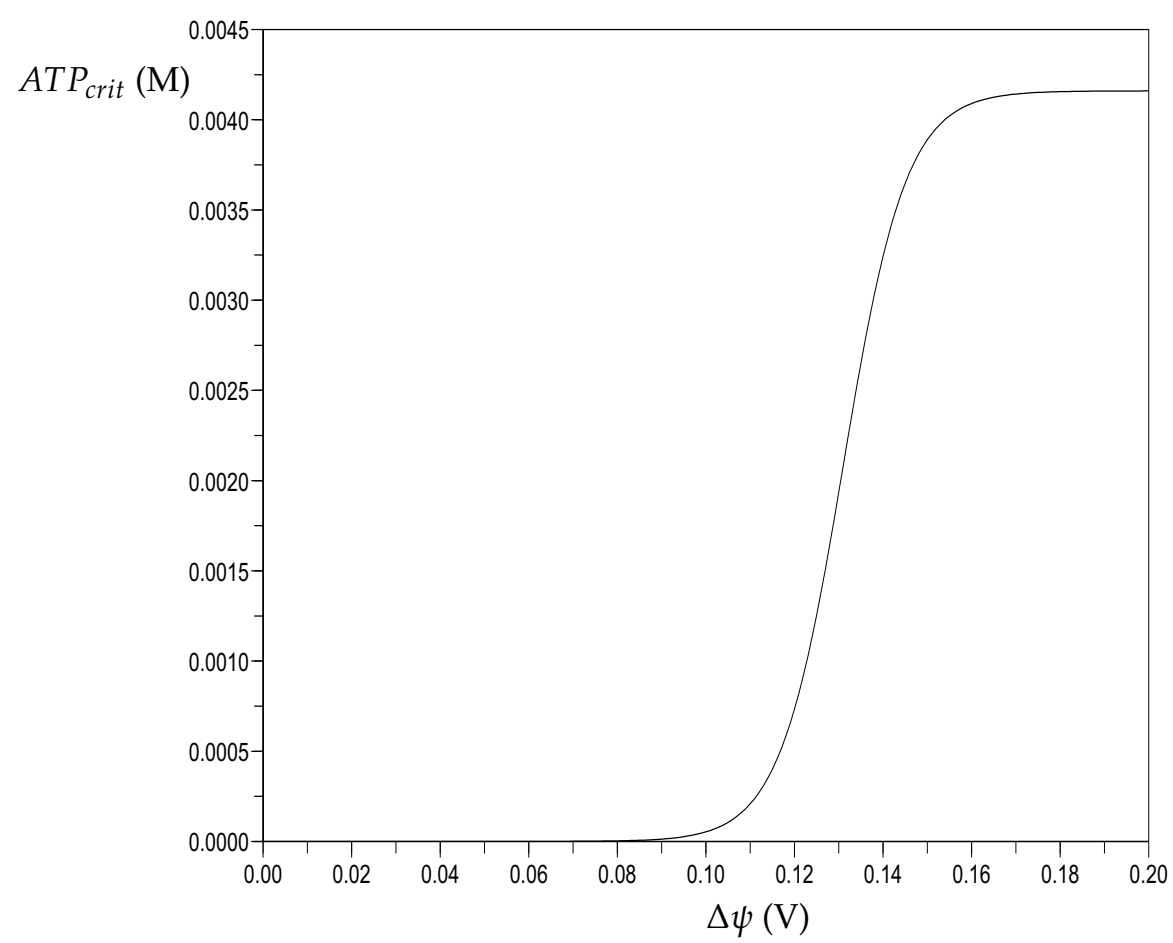

Figure 4: Critical threshold $A T P_{\text {crit }}$ as a function of $\Delta \psi$ values. $A T P_{\text {crit }}$ is the value of $A T P$ for which there is no synthesis or hydrolysis of ATP by ATP synthase. $A_{t}=4.16 \mathrm{mM}, R=8.314 \mathrm{~J}(\mathrm{~mol} \mathrm{~K})^{-1}$, $T=298 \mathrm{~K}, K_{a p p}=4.4 \mu \mathrm{M}, P i=2.44 \mathrm{mM}$.

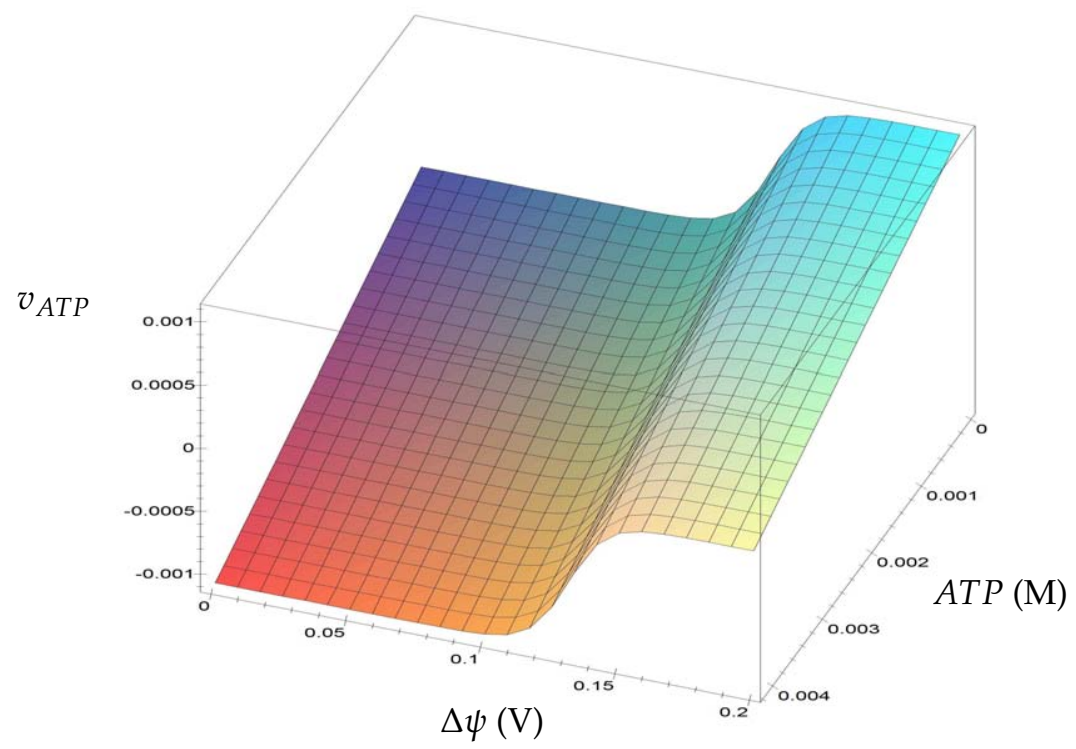

Figure 5: Rate of ATP synthase $v_{\text {ATP }} . k_{a t p}=131.9 \mathrm{mMs}^{-1}, b=4 \mathrm{M}^{-1}$. 
- The proton leak

$$
H_{e}^{+} \stackrel{v_{\text {leak }}}{\longrightarrow} H^{+}
$$

Because the inner mitochondrial membrane cannot be entirely impermeable to protons we introduce a $H^{+}$leak which is a function of $\Delta \psi$. We express this dependency by a simple linear relationship :

$$
v_{\text {leak }}=k_{\text {leak }} \Delta \psi
$$

\subsection{The differential equations governing the system}

The overall system is governed by the following set of 8 differential equations

$$
\begin{aligned}
\operatorname{Pyr}^{\prime}(t) & =v_{1}-v_{2}-v_{7} \\
\operatorname{AcC} A^{\prime}(t) & =v_{2}-v_{3} \\
\operatorname{Cit}^{\prime}(t) & =v_{3}-v_{4} \\
K G^{\prime}(t) & =v_{4}+v_{6}-v_{5} \\
O A A^{\prime}(t) & =v_{5}+v_{7}-v_{3}-v_{8}-v_{6} \\
N A D^{\prime}(t) & =-v_{2}-v_{4}-2 v_{5}+v_{\text {resp }} \\
A T P^{\prime}(t) & =v_{A T P}-v_{A N T}+v_{5}-v_{7} \\
\Delta \psi^{\prime}(t) & =\frac{1}{C}\left(10 v_{\text {resp }}-3 v_{A T P}-v_{\text {leak }}-v_{A N T}\right)
\end{aligned}
$$

with the following rate equations

$$
\begin{aligned}
& v_{1}=k_{1} \quad v_{2}=k_{2} \text { Pyr NAD } \\
& v_{3}=k_{3} O A A A c C o A \\
& v_{4}=k_{4} \operatorname{CitNAD} v_{5}=k_{5} \operatorname{KGNAD}\left(A_{t}-A T P\right) \quad v_{6}=k_{6}\left(O A A-\frac{K G}{K_{e q}}\right) \\
& v_{7}=k_{7} \text { Pyr ATP } v_{8}=k_{8} \text { OAA } \\
& v_{\text {leak }}=k_{\text {leak }} \Delta \psi \\
& v_{\text {resp }}(N A D, \Delta \psi)=k_{\text {resp }} \frac{N_{t}-N A D}{K+N_{t}-N A D} \frac{1}{1+\exp \left(a\left(\Delta \psi-\Delta \psi_{m}\right)\right)} \\
& v_{A T P}(A T P, \Delta \psi)=k_{A T P}\left(\frac{2}{1+\exp \left(b\left(A T P-A T P_{c r i t}(\Delta \psi)\right)\right)}-1\right) \\
& \operatorname{ATP}_{\text {crit }}(\Delta \psi) \quad= \\
& 1+\frac{\exp \left(\frac{-3 \Delta G_{\text {transport }}}{R T}\right)}{K_{a p p} P i}
\end{aligned}
$$

and a set of non-negative initial conditions

$$
\operatorname{Pyr}(0)>0, \operatorname{AcCo} A(0)>0, \cdots, \Delta \psi(0)>0
$$

where the parameters $k_{1}$ to $k_{8}, k_{\text {resp }}, k_{A T P}, k_{\text {leak }}, k_{A N T}, K_{\text {eq }}, K_{a p p}, C, K, \Delta \psi_{m}, a, b$ are constant and positive.

\subsection{The steady state hypothesis and parameters of the model}

In order to simulate actual experimental results, we used the Faraday constant $F=96485 \mathrm{C} / \mathrm{mol}$, the gas constant $R=8.314 \mathrm{~J} /(\mathrm{mol} \mathrm{K})$, and the absolute temperature $T=298 \mathrm{~K}$. During time intervals of several minutes and sometimes much more, it is experimentally observed that the concentrations of most of the metabolites are constant, which led us to look for stable stationary states mathematically and in the simulations. As regards the metabolite concentrations at steady state, we took the mean of several values found in the literature (between 10 and 20-see table 1) which presumably corresponds to a physiological intermediate state between a resting state (state 4: no ATP synthesis) and an active state (state 3: maximal ATP synthesis). This state has sometimes been called state 3.5 ([28]).

\footnotetext{
${ }^{1}$ The concentrations listed in table 1 are all given in $\mathrm{mM}$, whereas in the referenced articles they are often given in $\mu \mathrm{mole} /(\mathrm{g}$ fresh weight), $\mu \mathrm{mole} /(\mathrm{g}$ dry weight) or $\mu \mathrm{mole} /(\mathrm{g}$ protein). In order to have all those concentrations in mM, we collected values that gave us an idea of the converting factors: we used literature values for the ratio dry weight/fresh weight [55, 3, 23, 36, 5, 49, $9,45,52]$, cell volumes [34, 43, 39, 13, 47, 40, 7, 33], and values for the ratio cytoplasm volume/dry weight [45]. Even though these values were determined for different organisms, the average should be a good approximation. Conversion factors: $\mu \mathrm{mole} /(\mathrm{g}$ fresh weight) into mM: $1.19 ; \mu$ mole/(g dry weight) into mM: $0.35 ; \mu$ mole/(g protein) into mM: 0.25 . For some of the concentrations, when necessary, we used a mitochondrial volume/cytoplasm volume ratio of $0.18[45,52]$.
} 


\begin{tabular}{l}
\multicolumn{4}{|c}{ ACCEPTED MANUSCRIPT } \\
\begin{tabular}{|l|l|l|l|}
\hline species or rates & values in vivo & unit $^{1}$ & references \\
\hline$\overline{P y r}$ & 0.140 & $\mathrm{mM}$ & {$[11,45,17,37,25,56,31,52]$} \\
\hline$\overline{C i t}$ & 0.400 & $\mathrm{mM}$ & {$[6,11,17,25,45,52,56]$} \\
\hline$\overline{A c C o A}$ & 0.070 & $\mathrm{mM}$ & {$[11,17,19,45,37,52,38,54]$} \\
\hline$\overline{K G}$ & 0.250 & $\mathrm{mM}$ & {$[11,16,17,29,38,45,52]$} \\
\hline$\overline{O A A}$ & 0.005 & $\mathrm{mM}$ & {$[6,11,17,29,45,52,56]$} \\
\hline$\overline{N A D}$ & 0.940 & $\mathrm{mM}$ & {$[11,17]$} \\
\hline$\overline{A T P}$ & 3.230 & $\mathrm{mM}$ & {$[6,11,38]$} \\
\hline$\overline{\Delta \psi}$ & 150 & $\mathrm{mV}$ & {$[20,24]$} \\
\hline$\overline{v_{\text {resp }}}$ & 0.076 & $\mathrm{mM} / \mathrm{sec}$ & {$[4,42]$} \\
\hline$\overline{v_{A T P}}$ & 0.174 & $\mathrm{mM} / \mathrm{sec}$ & {$[4]$} \\
\hline
\end{tabular}
\end{tabular}

Table 1: Steady state concentration values at state 3.5

Some other concentrations and equilibrium constants (which are taken to be constant in the model) were also found in the literature (see table 2).

The constants used in $v_{\text {resp }}$ or $v_{A T P}, K=2 \mathrm{mM}, \Delta \psi_{m}=150 \mathrm{mV}, a=0.100 \mathrm{mV}^{-1}$ and $b=0.004 \mathrm{mM}^{-1}$

\begin{tabular}{|c|c|c|c|c|c|c|c|c|}
\hline species & asp & glu & $\mathrm{Pi}$ & $N_{t}$ & $A_{t}$ & $K_{a p p}$ & $K_{6, e q}{ }^{2}$ & C \\
\hline values in vivo & 1.600 & 5.300 & 2.440 & 1.070 & 4.160 & $4.4 e-6$ & 0.12 & $6.75 e-6$ \\
\hline unit & $\mathrm{mM}$ & $\mathrm{mM}$ & $\mathrm{mM}$ & $\mathrm{mM}$ & $\mathrm{mM}$ & dimensionless & dimensionless & $\mathrm{mM} / \mathrm{mV}$ \\
\hline references & $\begin{array}{l}{[11,17,45]} \\
{[52]}\end{array}$ & $\begin{array}{l}{[11,17,25]} \\
{[45,52,56]}\end{array}$ & {$[6,45]$} & {$[11,17]$} & $\begin{array}{l}{[6,11,45]} \\
{[38]}\end{array}$ & {$[2,22]$} & {$[1,14,45]$} & {$[21,51]$} \\
\hline
\end{tabular}

Table 2: Constants of the model

were chosen to fit experimental results. Then, the other parameters were calculated in order to fit the steady state concentrations or rates. $k_{1}$ to $k_{8}, k_{\text {resp }}, k_{A T P}, k_{\text {leak }}, k_{A N T}$ are rate constants. As we did not have experimental values for $v_{\text {leak }}$ and $v_{A N T}$, we decided to fix $k_{1}$ and $k_{3}$ in order to obtain the same order of magnitude for all the rates at steady state. In order to obtain for the steady state the values given in table 1 , we calculated the other parameters as follows (where $\bar{x}$ denoted the $x$ concentration at steady state)

$$
\begin{aligned}
& k_{2}=\frac{\overline{O A A} \overline{A c C o A}}{\overline{P y r} \overline{N A D}} k_{3} \\
& k_{4}=\frac{\overline{O A A} \overline{A c C O A}}{\overline{C i t} \overline{N A D}} k_{3} \\
& k_{6}=\frac{\overline{v_{\text {resp }}}-4 \overline{O A A} \overline{A c C o A} k_{3}}{2\left(\overline{O A A}-\frac{\overline{K G}}{K_{e q}}\right)} \\
& k_{7}=\frac{k_{1}-\overline{O A A} \overline{A c C o A} k_{3}}{\overline{P y r} \overline{A T P}} \\
& k_{5}=\frac{\overline{v_{\text {resp }}}-2 \overline{O A A} \overline{A c C o A} k_{3}}{2 \overline{K G} \overline{N A D}\left(A_{t}-\overline{A T P}\right)} \\
& k_{8}=\frac{k_{1}-\overline{O A A} \overline{A c C o A} k_{3}}{\overline{O A A}} \\
& \begin{aligned}
k_{A T P} & =\frac{\overline{v_{\text {ATP }}}}{\left(\frac{2}{1+\exp \left(b\left(\overline{A T P}-A T P_{\text {crit }}(\overline{\Delta \psi})\right)\right.}-1\right)} \\
k_{A N T} & =\frac{2 \overline{v_{A T P}}+\overline{v_{\text {resp }}}-2 k_{1}}{2 \overline{A T P}}
\end{aligned} \\
& \begin{aligned}
k_{\text {resp }}= & \frac{\overline{N_{t}-\overline{N A D}} \frac{\overline{v_{\text {resp }}}}{K+N_{t}-\overline{N A D}} \frac{1}{1+\exp \left(a\left(\overline{\Delta \psi}-\Delta \psi_{m}\right)\right)}}{k_{\text {leak }}=} \\
& \frac{10 \overline{v_{\text {resp }}}-3 \overline{v_{A T P}}-\overline{v_{A N T}}}{\overline{\Delta \psi}}
\end{aligned}
\end{aligned}
$$

As the values of the concentrations at steady state vary from 1 to 1000 , the parameters of the model are not of the same order of magnitude. For this reason, the variables and the time are scaled according to

$$
\begin{array}{llll}
p=\frac{P y r}{\overline{P y r}} & a=\frac{A c C o A}{\overline{A c C o A}} & c=\frac{C i t}{\overline{C i t}} & k=\frac{K G}{\overline{K G}} \\
o=\frac{O A A}{\overline{O A A}} & n=\frac{N A D}{N_{t}} & e=\frac{A T P}{A_{t}} & s=\frac{\Delta \psi}{\Delta \psi_{m}} \\
\tau=\frac{k_{1}}{\overline{P y r}} t &
\end{array}
$$

\footnotetext{
${ }^{2}$ The equilibrium constant of reaction 6 given here is defined as $K_{6, e q}=\frac{K G_{e q} A s p_{e q}}{O A A_{e q} G l u_{e q}}$. From this value and the values for as $p$ and $g l u$, our equilibrium constant was calculated $K_{e q}=K_{6, e q} \frac{G l u_{e q}}{A s p_{e q}}$.
} 
which leads to a new system (31-38) (see Annex).

\section{Theoretical results and numerical simulations}

Lemma 3.1 For each set of non-negative initial conditions, the system (16-23) admits a globally defined solution on $[0, \infty)$. Each of the solution components (except $\Delta \psi)$ remains non-negative for all $t>0$. It also verifies the constraints $N A D<N_{t}$ and $A T P<A_{t}$.

Lemma 3.2 The system (16-23) admits at least one non-trivial steady state

$$
\bar{S}=(\overline{P y r}, \overline{A c C o A}, \overline{C i t}, \overline{K G}, \overline{O A A}, \overline{N A D}, \overline{A T P}, \overline{\Delta \psi}) .
$$

Proof of lemma 3.1 and 3.2

This follows from the transformation of system (16-23) to system (31-38) combined with lemma 6.1 and 6.2 (see annex).

Numerically, for any set of parameters and initial conditions, the system seems to have a unique global asymptotic steady state. This is shown in the following three figures (fig. 6-8). In fig. 6, the initial conditions are chosen at $10 \%$ away from the steady state. The steady state is rapidly reached after some damped oscillations. Figure 7 depicts the case of low ATP concentration. This is a typical experimental situation when $A D P$ is added to a suspension of mitochondria. ATP is synthesized at the expense of $\Delta \psi$ which drops rapidly, then recovers in the same time, as ATP is regenerated. This is exactly what is experimentally observed in these types of experiments. Usually the variations in concentration of TCA cycle metabolites are not recorded, so the single oscillation generated in this simulation has not yet been observed to our knowledge. However, it can easily be understood as the consequence of the fast decrease in ATP concentration. Then it propagates through $\triangle \psi, N A D H$ and the respiratory chain, and finally through $N A D H$ to the TCA cycle. It should be noticed, however, that most of the variations in TCA metabolites are (very) weak. In fig. 8, the ATP concentration is higher than its steady state value so $A T P$ hydrolyzes in $A D P$ extruding protons and increasing $\Delta \psi$. Then $\Delta \psi$ returns slowly to its steady state value. Due to the increase in $\Delta \psi$ the rate of respiration is decreased, as deduced from the decrease in NAD concentration. All these changes are accompanied by small damped oscillations of TCA cycle metabolites.

The parameters determined previously in section 2.4 for an intermediate state of respiration have been used to simulate low and high ATP synthesis. For this aim $k_{A N T}$ was varied keeping all other parameters constant. $k_{A N T}$ deals with ATP/ADP carrier but can also represent the intensity of cell ATP consumption $\left(v_{A N T}=k_{A N T} A T P\right)$. The results are represented in table 3 . When $k_{A N T}$ is decreased (towards state 4) we observe an increase in ATP concentration, a decrease in NAD concentration, in respiration $\left(v_{\text {resp }}\right)$ and in $v_{A T P}$. The reverse occurs when $k_{A N T}$ is increased. The range of responses - from 183 to $138 \mathrm{mV}$ for $\Delta \psi$, from 3.98 to $2.22 \mathrm{mM}$ for ATP, from 23.6 to $92.5 \mu \mathrm{Ms}^{-1}$ for $v_{\text {resp }}$ and from 46.1 to $214.5 \mu \mathrm{Ms}^{-1}$ for $v_{\text {ATP }}$ are in good agreement with the values of table II in [12].

In order to evaluate the effect of the reversibility of the other reactions, we have introduced a new term in the equations of $v_{3}$ and $v_{7}$ which are now:

$$
v_{3}=k_{3}\left(\mathrm{OAAAcCoA}-\frac{C i t}{K_{3, e q}}\right) \quad v_{7}=k_{7}\left(\operatorname{Pyr} A T P-\frac{\left(A_{t}-A T P\right) O A A}{K_{7, e q}}\right)
$$

where $K_{3, e q}=\frac{1 e 6}{\operatorname{CoASH}_{e q}}([30]), K_{7, e q}=\frac{1}{P i_{e q}}([30])$,

with $\mathrm{CoASH}_{e q} \approx 10^{-3} \mathrm{mM}$ ([45]). With these new reversible equations we show that we are still able to obtain the steady state corresponding to the physiological concentrations of TCA-cycle metabolites and to the intermediate rate of respiration, ATP synthesis and transmembrane potential summarised in table 1. With this model it is still possible to reach states 4 and 3 and the results are the same as in table 3.

\section{Discussion}

This simple model accounts at least qualitatively for the salient features of the oxidative phosphorylation system, namely:

- the coupling between the ATP synthesis and the respiratory chain and the coupling between the respiratory chain and the TCA cycle. When there is a demand in ATP (low ATP in fig. 7), ATP is 


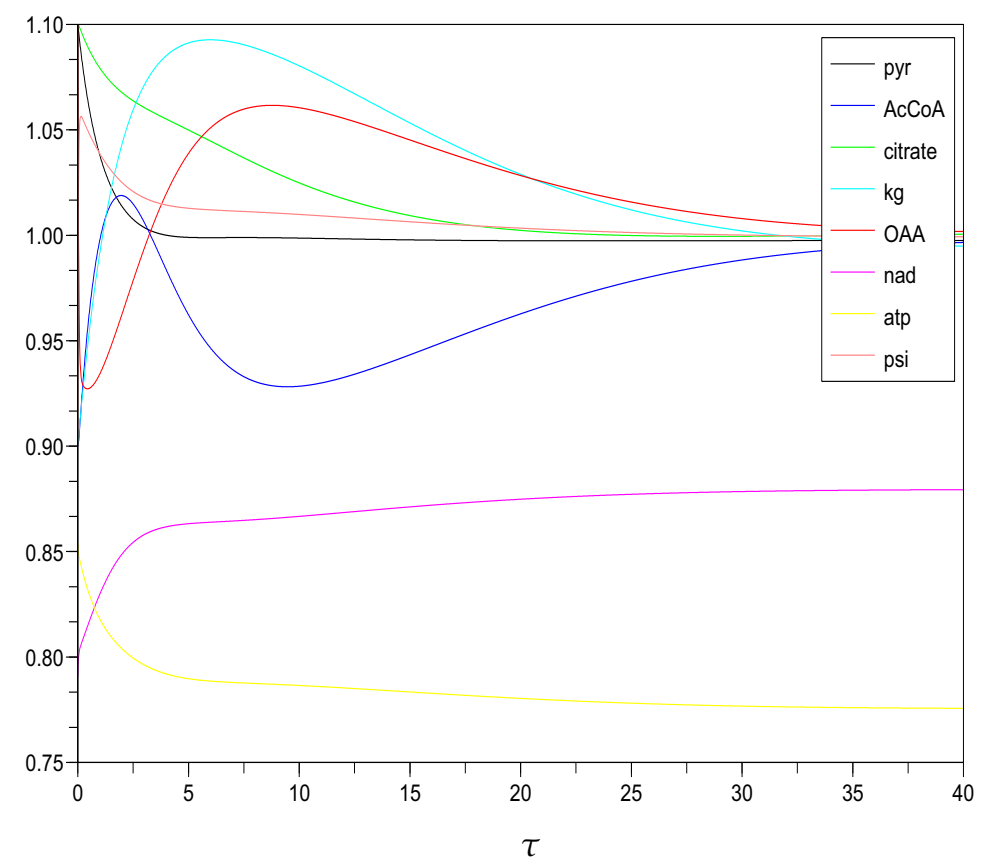

Figure 6: Metabolite concentrations time course towards the steady state (initial conditions are taken as $\pm 10 \%$ away from the steady state)with the following parameters:

$\beta_{2} \approx 0.60, \beta_{3} \approx 0.53, \beta_{4} \approx 0.60, \beta_{5} \approx 2.41, \beta_{6} \approx=0.42 e-3, \beta_{7} \approx 0.61, \beta_{8} \approx 0.47, \beta_{\text {ANT }} \approx 5.90, \beta_{\text {leak }} \approx 1.68$, $\beta_{\text {resp }} \approx 65.54, \beta_{\text {ATP }} \approx 3470.90, \delta_{6} \approx 125.78, \delta_{r 1} \approx 1.87, \delta_{r 2} \approx 15, \delta_{\text {atp }} \approx 0.016, \delta_{\text {crit }} \approx 21.02, K_{\text {app }}^{\prime}=1.05 e-8$.

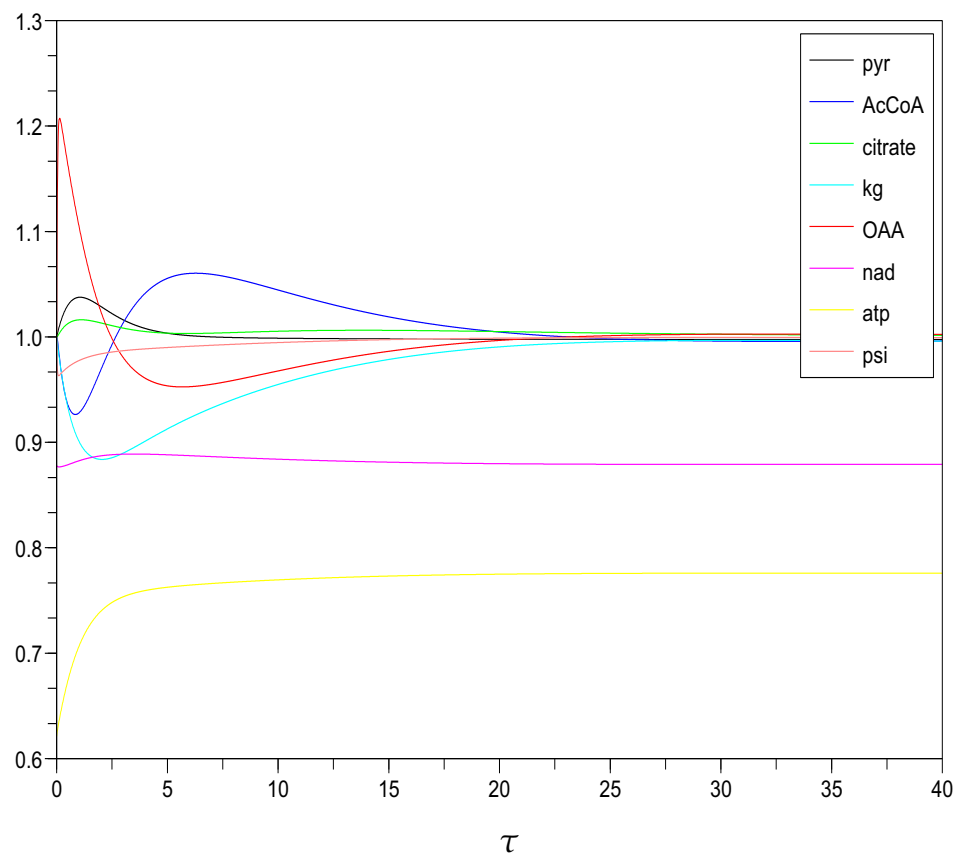

Figure 7: Low ATP initial concentration (other initial values are taken as their steady state values). 


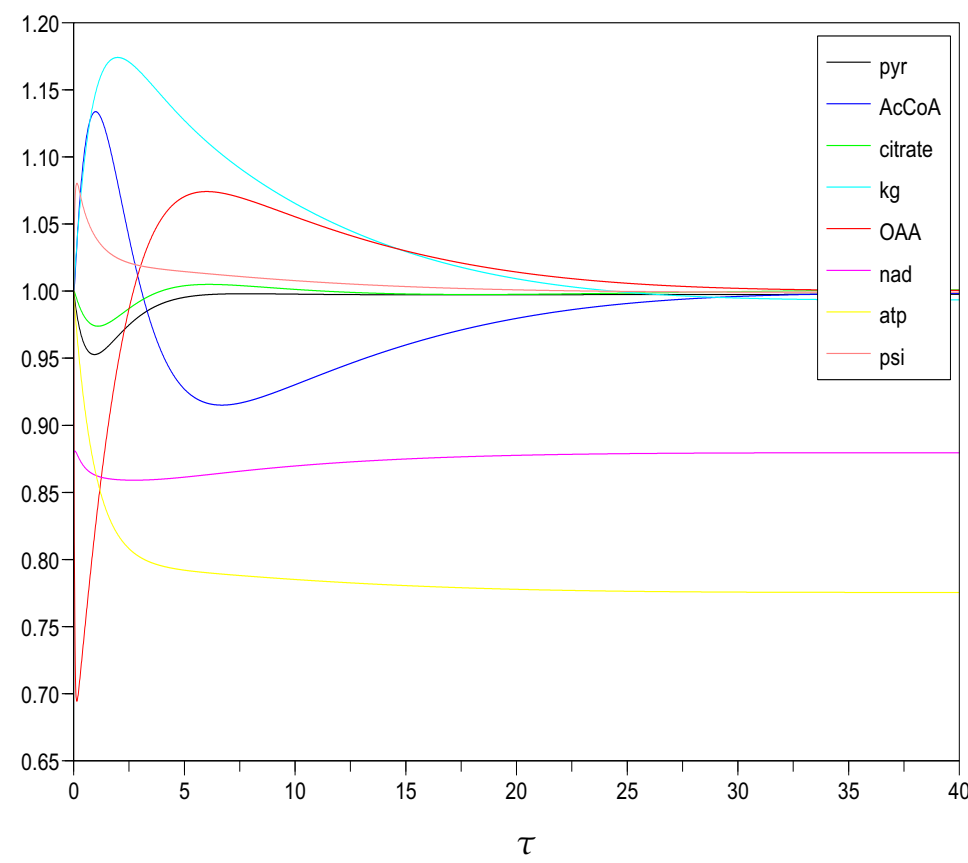

Figure 8: High ATP initial concentration (other initial values are taken as their steady state values).

\begin{tabular}{|l|l|l|l|}
\hline species or rates & state 4 & state 3.5 & state 3 \\
\hline$k_{A N T}$ & 0.1 & 0.05387 & 0.005 \\
\hline$\overline{P_{y r}}$ & 0.161 & 0.140 & 0.185 \\
\hline$\overline{\text { Cit }}$ & 0.460 & 0.400 & 0.528 \\
\hline$\overline{A c C o A}$ & 0.105 & 0.070 & 0.019 \\
\hline$\overline{K G}$ & 0.146 & 0.250 & 0.693 \\
\hline$\overline{O A A}$ & 0.004 & 0.005 & 0.008 \\
\hline$\overline{N A D}$ & 0.968 & 0.940 & 0.309 \\
\hline$\overline{A T P}$ & 2.227 & 3.230 & 3.982 \\
\hline$\overline{\Delta \psi}$ & 138 & 150 & 183 \\
\hline$\overline{v_{2}}=\overline{v_{3}}=\overline{v_{4}}$ & 23.7 & 20 & 8.7 \\
\hline$\overline{v_{5}}$ & 22.5 & 18 & 3.1 \\
\hline$\overline{v_{6}}$ & -1.2 & -2 & -5.6 \\
\hline$\overline{v_{7}}=\overline{v_{8}}$ & 14.3 & 18 & 29.3 \\
\hline$\overline{v_{l}}$ & 58.9 & 64 & 78.2 \\
\hline$\overline{v_{A N T}}$ & 222.7 & 174 & 19.9 \\
\hline$\overline{v_{\text {resp }}}$ & 92.5 & 76 & 23.6 \\
\hline$\overline{v_{A T P}}$ & 214.5 & 174 & 46.1 \\
\hline
\end{tabular}

Table 3: Steady state concentration values (in $\mathrm{mM}$ ) and rates (in $\mu \mathrm{M} / \mathrm{s}^{-1}$ ) at states $4,3.5$ and 3 . with the following parameters:

$k_{1}=38 \mu \mathrm{Ms}^{-1}, k_{2}=152, k_{3}=57142, k_{4}=53, k_{7}=40$ in $(\mathrm{M} \mathrm{s})^{-1}, k_{5}=82361 \mathrm{M}^{-2} \mathrm{~s}^{-1}, k_{6}=3.2 e-3, k_{8}=3.6$ in $\mathrm{s}^{-1}, k_{\text {resp }}=2.5 k_{\text {atp }}=131.9 \mathrm{mMs}^{-1}, k_{l}=0.426 \mathrm{M}(\mathrm{mVs})^{-1}$. 
synthesized at the expense of $\Delta \psi$. The decrease in $\Delta \psi$ leads to an increase in the rate of respiration, which increases the demand in NADH supply by the TCA cycle.

- On the contrary, an excess of $A T P$ is hydrolyzed, increasing the $\Delta \psi$ and decreasing the rate of respiration and the TCA cycle turn-over. This situation is encountered experimentally in cells lacking mitochondrial DNA ( $\rho^{0}$ cells) ([15]).

- The steady state appears numerically unique and stable, as is the case in the in vivo system. However, the variations seem more rapid than in the in vivo system. This could be due to the absence of other ions than protons giving rise to coupled exchange of ions slowing down all the processes. It could also be due to the absence of buffers of these ions (including protons) in our model. It is well known that the polar parts of phospholipids and proteins can bind ions. Special proteins can even complex some specific ions like calcium. The buffering power of ions might smooth and slow down the variations, particularly the damped oscillations.

However several simplifying assumptions at the basis of the model can be questioned.

- The use of mass action kinetics is a very strong simplification. It has the great advantage of making an analytical processing possible, but one can argue that saturation is a key feature of biological reactions. We have nevertheless introduced a hyperbolic kinetic for NADH dehydrogenase (in $v_{\text {resp }}$ ) because it is the main output flux. This is clear from table 3 in which $v_{\text {resp }}$ is varied from a low value to a value close to its maximum. At the same time the rates in TCA cycle vary reasonably, between 8.7 and 23.9 for $v_{2}=v_{3}=v_{4}$ and between 3.1 and 22.5 for $v_{5}$.

- Irreversible reactions and regulations

The main reason for not introducing reversible reactions or regulation was the same as above, i.e. to work with a mathematically tractable model. As our aim was to obtain a simple model describing the links between the variations of TCA cycle when the oxidative phosphorylations are varied according to different cell ATP demands, we took as simple equations as possible. It means that we did not introduce regulations and took irreversible reactions even for some reversible reactions, but which always have the same direction in physiological conditions. This is the case for example of pyruvate carboxylase which is reversible but "formation of pyruvate from oxaloacetate by reversal of this process is unlikely since the intracellular concentration of oxaloacetate is very small" ([30]). We take great care in our fitting procedure to start with very low oxaloacetate concentrations (see table 1) and we checked that this concentration remains low in our simulation (table 3). Reaction 5, which is taken as irreversible, is in fact partially reversible because oxaloacetate can be converted into $\alpha$-ketoglutarate by reaction 6 . In order to evaluate the effect of the reversibility of the other reactions, we have introduced a new term in the equation of $v_{3}$ and $v_{7}$. With these new reversible equations it is still possible to obtain the steady state corresponding to the physiological concentrations of TCA-cycle metabolites and to an intermediate rate of respiration, ATP synthesis and transmembrane potential listed in table 1. With this model it is possible to reach states 4 and 3 (same results as in table 3). A reversible model has the advantage of exhibiting some kind of regulation due to the reversible inhibitory effect of the products. In addition, a reversible model can be particularly useful to describe the effect of mutations that occur in mitochondrial diseases with accumulation of intermediate metabolites.

\section{Conclusion}

A simple model is useful for several reasons. First, because in many instances it is not necessary to cope with all the complexity of a biological system. For instance, in many models dealing with the mitochondrial synthesis of $A T P$, it is enough to know that this synthesis is coupled through the transmembrane potential $\Delta \psi$ to an increase in the rate of oxygen consumption and an acceleration of the TCA cycle. The coupling is more important to model than all the details of the ATP synthesis or the oxygen consumption themselves.

Secondly, the salient features of a system and their interrelationships are more apparent on a simple system. Here the salient features are the shape of $v_{\text {resp }}$ as a function of $\Delta \psi$, which is the result of several complex mechanisms. In our model the interrelationships (coupling) between the three large sets of reactions, ATP synthesis, rate of oxygen consumption and TCA cycle are more evident because each block is summarized in one or few reactions.

Another advantage of a very simple model is that it is more easily tractable from the mathematical point of view. However for mathematical studies we still have to deal with a quite large system and for this reason we transformed it into a planar system. By this way, we managed to prove the existence of at least one steady state. Biologically and numerically it seems that the steady state is unique and is a global attractor 
for the system. However some basic techniques of qualitative stability analysis, which are sometimes useful for large systems, fail with our model (see remark at the end of the annex). Moreover, we chose a dimensionless system among the several possible ones and there are very different time scales (a very fast transient for $\Delta \psi$ ). Before applying a singular perturbation method, the system needs to be improved because the variations in $\Delta \psi$ are too rapid. In this paper, we have presented a very simple model which remains difficult to analyse mathematically. However from the experimental point of view, we are tempted to add several features (reversibility of some reactions, regulations, etc.) to this model to take better the observed behaviours into account. The question then arises: until which point a simple model can be complexified? Where can the cursor be put? The answer will depend on the biological question to which the model is intended to respond, but it must be kept in mind that very simple models are nevertheless able to exhibit many salient features of the biological reality.

\section{Annex}

\subsection{Dimensionless system}

With the new scaled variables, the rate equations become

$$
\begin{aligned}
& v_{1}=1 \quad v_{2}=\beta_{2} p n \quad v_{3}=\beta_{3} \circ a \quad v_{4}=\beta_{4} c n \quad v_{5}=\beta_{5} k n(1-e) \\
& v_{6}=\beta_{6}\left(o-\delta_{6} k\right) \quad v_{7}=\beta_{7} p e \quad v_{8}=\beta_{8} o \quad v_{A N T}=\beta_{A N T} e \quad v_{\text {leak }}=\beta_{\text {leak }} s \\
& v_{\text {resp }}=\beta_{\text {resp }} \frac{1-n}{\left(\delta_{r 1}+1-n\right)} \frac{1}{1+\exp \left(\delta_{r 2}(s-1)\right)} \\
& v_{A T P}=\beta_{A T P}\left(\frac{2}{1+\exp \left(\delta_{\text {atp }}\left(e-e_{\text {crit }}(s)\right)\right)}-1\right) \\
& e_{\text {crit }}=\frac{K_{a p p}^{\prime}}{K_{a p p}^{\prime}+\exp \left(-\delta_{c r i t} s\right)}
\end{aligned}
$$

where the normalized constants are

$$
\begin{array}{llllll}
\beta_{2}=\frac{k_{2}}{k_{1}} N_{t} \overline{P y r} & \beta_{3}=\frac{k_{3}}{k_{1}} \overline{O A A A C C} & \beta_{4}=\frac{k_{4}}{k_{1}} N_{t} \overline{C i t} & \beta_{5}=\frac{k_{5}}{k_{1}} N_{t} A_{t} \overline{K G} & \beta_{6}=\frac{k_{6}}{k_{1}} \overline{O A A} & \beta_{7}=\frac{k_{7}}{k_{1}} A_{t} \overline{P y r} \\
\beta_{8}=\frac{k_{8}}{k_{1}} \overline{\mathrm{OAA}} & \beta_{A N T}=\frac{k_{A N T}}{k_{1}} A_{t} & \beta_{\text {leak }}=\frac{k_{\text {leak }}}{k_{1}} \Delta \psi_{m} & \beta_{\text {resp }}=\frac{k_{\text {resp }}}{k_{1}} & \beta_{A T P}=\frac{k_{A T P}}{k_{1}} & \\
\delta_{6}=\frac{\overline{K G}}{\overline{O A A} K_{\text {eq }}} & \delta_{r 1}=\frac{K}{N_{t}} & \delta_{r 2}=a \Delta \psi_{m} & \delta_{\text {atp }}=b A_{t} & \delta_{\text {crit }}=3 \frac{1.2 F \Delta \psi_{m}}{R T} & K_{\text {app }}^{\prime}=K_{\text {app }} P i
\end{array}
$$

The set of differential equations expressing the variation of the scaled variables is

$$
\begin{aligned}
\frac{d p}{d \tau} & =v_{1}-v_{2}-v_{7} \\
\epsilon_{1} \frac{d a}{d \tau} & =v_{2}-v_{3} \\
\epsilon_{2} \frac{d c}{d \tau} & =v_{3}-v_{4} \\
\epsilon_{3} \frac{d k}{d \tau} & =v_{4}+v_{6}-v_{5} \\
\epsilon_{4} \frac{d o}{d \tau} & =v_{5}+v_{7}-v_{3}-v_{8}-v_{6} \\
\epsilon_{5} \frac{d n}{d \tau} & =-v_{2}-v_{4}-2 v_{5}+v_{\text {resp }} \\
\epsilon_{6} \frac{d e}{d \tau} & =v_{A T P}-v_{A N T}+v_{5}-v_{7} \\
\epsilon_{7} \frac{d s}{d \tau} & =10 v_{\text {resp }}-3 v_{A T P}-v_{\text {leak }}-v_{A N T}
\end{aligned}
$$

with

$$
\epsilon_{1}=\frac{\overline{A c C o A}}{\overline{P y r}} \quad \epsilon_{2}=\frac{\overline{C i t}}{\overline{P y r}} \quad \epsilon_{3}=\frac{\overline{K G}}{\overline{P y r}} \quad \epsilon_{4}=\frac{\overline{O A A}}{\overline{P y r}} \quad \epsilon_{5}=\frac{N_{t}}{\overline{P y r}} \quad \epsilon_{6}=\frac{A_{t}}{\overline{P y r}} \quad \epsilon_{7}=\frac{\Delta \psi_{m}}{\overline{P y r} C}
$$




\subsection{Mathematical results}

Lemma 6.1 For each set of non-negative initial conditions, the system (31-38) admits a globally defined solution on $[0, \infty)$. Each of the solution components (except s) remains positive for all $t>0$. It also verifies the constraints $n<1$ and $e<1$.

Proof.

Local existence and uniqueness on some maximal time interval is straightforward by using the CauchyLipschitz theorem. Since the initial conditions are non-negative, and the vector field defined by the right hand side of (31-37) at the boundary of the region $\mathbb{R}_{+}^{7}$ never points outwards, all the concentrations $p, \cdots, n$ remain non-negative for all $\tau$.

If $e=1$ then according to the dimensionless rate equations (29), we have $e>e_{\text {crit }}$ and then $v_{\text {ATP }}<0$. Since $\epsilon_{6} \frac{d e}{d \tau}=v_{A T P}-v_{A N T}-\beta_{7} p<0$, it follows that $e$ decreases. Consequently, if $0<e(0)<1$ we have $0<e<1$.

By the same reasoning, we show that $n<1$ because $v_{\text {resp }}(1, s)=0$.

Set $S=p+\epsilon_{1} a+\epsilon_{2} c+\epsilon_{3} k+\epsilon_{4} o$, since $\frac{d S}{d \tau}=1-v_{3}-v_{8}$, we have $S(\tau)<S(0)+\tau$.

For $s$, we have $\epsilon_{7} \frac{d s}{d \tau}=f(\tau)-\beta_{\text {leak }} s$ where $f(\tau)=10 v_{\text {resp }}-3 v_{\text {ATP }}-v_{A N T}$ is bounded.

So $\epsilon_{7} s(\tau)=s(0) e^{-\beta_{\text {leak }} \tau}+\int_{0}^{\tau} f(u) e^{-\beta_{\text {leak }}(\tau-u)} d u$ and we obtain $\left|\epsilon_{7} s(\tau)\right| \leq s(0) e^{-\beta_{\text {leak }} \tau}+M$.

So $S$ and $s$ cannot be infinite in finite time. Therefore solutions of the system exist globally in time.

Lemma 6.2 There is at least one non-trivial steady state $\bar{S}=(\bar{p}, \bar{a}, \bar{c}, \bar{k}, \bar{o}, \bar{n}, \bar{e}, \bar{s})$ for the system (31-38).

Proof.

From (31), we derive at steady state

$$
\bar{p}=\frac{1}{\beta_{2} n+\beta_{7} e} .
$$

Adding the right hand side of (32) and (33), we obtain

$$
\bar{c}=\frac{\beta_{2}}{\beta_{4}\left(\beta_{2} n+\beta_{7} e\right)} .
$$

Similarly, with (33)-(34) and (35), we have

$$
\bar{o}=\frac{\beta_{7} e}{\beta_{8}\left(\beta_{2} n+\beta_{7} e\right)} .
$$

With (31) to (35), we find

$$
\bar{a}=\frac{\beta_{2} \beta_{8} n}{\beta_{3} \beta_{7} e}
$$

from (34),

$$
\left.\bar{k}=\frac{\left(\beta_{2} \beta_{8} n+\beta_{6} \beta_{7} e\right)}{\beta_{8}\left(\beta_{2} n+\beta_{7} e\right)\left(\beta_{6} \delta_{6}+\beta_{5} n(1-e)\right.}\right)
$$

and adding $(36)-\frac{1}{10}(38)-\frac{3}{10}(37)$, we obtain

$$
\bar{s}=\frac{10}{\beta_{\text {leak }}}\left(2 \beta_{2} \bar{p} n+\frac{23}{10} \beta_{5} \bar{k} n(1-e)-\frac{4 \beta_{A N T}}{10} e-\frac{3}{10} \beta_{7} \bar{p} e\right) .
$$

Therefore the existence of a steady state is related to the existence of $(n, e)$ satisfying $\left(f_{1}, f_{2}\right)(n, e)=(0,0)$ with

$$
f_{1}(n, e)=-\beta_{2} \bar{p} n-\beta_{4} \bar{c} n-2 \beta_{5} \bar{k} n(1-e)+v_{r e s p}
$$

and

$$
f_{2}(n, e)=v_{A T P}-v_{A N T}+\beta_{5} \bar{k} n(1-e)-\beta_{7} \bar{p} e .
$$

Now we prove that such $(n, e)$ exists. Let us define the function $F=\left(f_{1}, f_{2}\right)$. Since $f_{1}(0, e)=v_{\text {resp }}>0$, $f_{1}(1, e)<0, f_{2}(n, 0)=v_{A T P}(n, 0)>0$ and $f_{2}(n, 1)=v_{A T P}(n, 1)-k_{A N T}-\beta_{7} p<0$, then $F$ vanishes at least once on $] 0,1[\times] 0,1[$. In order to verify this, we apply lemma 6.3 . 
Lemma 6.3 If $F=\left(f_{1}, f_{2}\right): \mathbb{R}^{2} \leftarrow \mathbb{R}^{2}$ is a continuous vector field such that

$$
\begin{array}{lll}
f_{1}(0, e)>0 & f_{1}(1, e)<0 & \forall e \in[0,1] \\
f_{2}(n, 0)>0 & f_{2}(n, 1)<0 & \forall n \in[0,1]
\end{array}
$$

then there exists $\bar{m}=(\bar{n}, \bar{e})$ which satisfies

$$
0<\bar{n}<1, \quad 0<\bar{e}<1, \quad F(\bar{m})=0
$$

Proof.

Let's note the square $C=\left\{(n, e) \in \mathbb{R}^{2} ; 0<n<1, \quad 0<e<1\right\}$ and define the following homotopy

$$
H^{\tau}(n, e)=(1-\tau) F(n, e)+\tau G(n, e) \quad \tau \in[0,1]
$$

with $G(n, e)=\left(\frac{1}{2}-n, \frac{1}{2}-e\right)$. From the assumption on $F$, for all $\tau \in[0,1], F$ never vanishes on $\partial C$. We can define the degree of $H^{\tau}$ and using homotopy invariance we have

$$
\operatorname{deg}\left(H^{\tau}, C, 0\right)=\operatorname{deg}\left(H^{0}, C, 0\right)=\operatorname{deg}\left(H^{1}, C, 0\right) .
$$

As $H^{1}=G$ and $G$ vanishes only on $\left(\frac{1}{2}, \frac{1}{2}\right)$, we obtain

$$
\operatorname{deg}\left(H^{1}, C, 0\right)=\operatorname{sign}\left(\operatorname{det}\left(J_{G}\left(\frac{1}{2}, \frac{1}{2}\right)\right)\right)=1 \neq 0
$$

where $\operatorname{sign}\left(\operatorname{det}\left(J_{G}\left(\frac{1}{2}, \frac{1}{2}\right)\right)\right)$ is the sign of the determinant of the jacobian of $G$ at $\left(\frac{1}{2}, \frac{1}{2}\right)$. Since the degree of $H^{0}=F$ is not equal to zero, $F$ vanishes at leat once inside $C$ ( $\S$ A chapter 12 in [46]).

Remark :

If $F$ is not continuous at $(0,0)$, the same result holds by applying the same argument to the function on the smaller square $[\epsilon, 1] \times[\epsilon, 1]$.

Remark:

Let us denote $J=\left(a_{i j}\right)_{1 \leq i, j \leq 8}$ the jacobian at steady state. The system is not qualitatively stable (because $a_{i j} a_{j i}$ can be positive for some $(i, j)$ i.e the members of some given interacting species do not have opposite effects on each other). However it seems that the magnitudes of interaction are appropriately balanced. Unfortunately, $J$ is not also diagonally dominant (i.e there are not $n$ numbers $d_{n}>0$ such that $\left.a_{i i} d_{i}+\sum_{j \neq i}\left|a_{i j}\right| d_{j}<0 \forall i=1, \cdots, n\right)$.

\section{Acknowledgements}

We thank A. Ducrot and J.F. Bony (IMB) for their helpful discussion. We are indebted to Ray Cooke for revising the english. This work was supported by ACI IMPBio and ANR sysBio 2007.

\section{References}

[1] Alberty R. A., 1998. Calculation of Standard Transformed Formation Properties of Biochemical Reactants and Standard Apparent Reduction Potentials of Half Reactions, Arch. Bioch. Biophys., Vol. 358, No 1, 25-39.

[2] Alberty, R., 2000. Calculating apparent équilibrium constants of enzyme-catalyzed reactions at $\mathrm{pH}$ 7, Bioch. Education 28, 12-17.

[3] Andersen, J. B., Rourke B. C., Caiozzo, V. J., Bennett A. F., Hicks J. W., 2005. Physiology: postprandial cardiac hypertrophy in pythons. Nature 434, 37-38.

[4] Anflous, K., Veksler, V., Mateo, P., Samson, F., Saks, V., Ventura-Clapier, R., 1997. Mitochondrial creatine kinase isoform expression does not correlate with its mode of action. Biochem. J. 322, 7378.

[5] Arai, K., Lee, K., Berthiaume, F., Tompkins, R. G., 2001. Intrahepatic amino acid and glucose metabolism in a D-galactosamine-induced rat liver failure model. Hepatology 34(2), 360-361.

[6] Armson, A., Mendis, A. H., 1995. Steady state content of glycolytic/tricarboxylic acid-cycle intermediates, adenine nucleotide pools and the cellular redox-status in the infective (L3) larvae of (homogonic) Strongyloides ratti. Int. J. Parasitol. 25(2), 197-202. 
[7] Attene-Ramos, M. S., Kitiphongspattana, K., Ishii-Schrade, K., Gaskins H. R., 2005. Temporal changes of multiple redox couples from proliferation to growth arrest in IEC-6 intestinal epithelial cells. Am. J. Physiol. Cell Physiol. 289(5), C1220-C1228.

[8] Atkinson, D.E., 1990. What should a theory of metabolic control offer to the experimenter ? in Control of Metabolic Processes, edited by Cornish-Bowden, A. and Cardenas, M.L., Springer, 3-11.

[9] Bagshaw, A. P., Farquharson, M. J., 2002. Simultaneous determination of iron, copper and zinc concentrations in skin phantoms using XRF spectrometry. X-Ray Spectrometry 31(1), 47-52.

[10] Beard, D.A., 2005. A biophysical model of the mitochondrial respiratory system and oxidative phosphorylation. Plos Comp. Biol. 1, 252-264.

[11] Bergmeyer, H.U., 1974. Methods of enzymatic analysis. Verlag Chem. Int., Weinheim.

[12] Bohnensack, R., 1981. Control of energy transformation of mitochondria. Analysis by a quantitative model. Biochim. Biophys. Acta 634, 203-218.

[13] Chin, J., Miller, S. C., Wada, M., Nagano, N. Nemeth, E. F., Fox, J., 2000. Activation of the calcium receptor by a calcimimetic compound halts the progression of secondary hyperparathyroidism in uremic rats. J. Am. Soc. Nephrol. 11, 903-911.

[14] Cortassa, S., Aon, M. A., Marbán, E., Winslow, R. L., O’Rourke, B., 2003. An integrated model of cardiac mitochondrial energy metabolism and calcium dynamics. Biophys. J. 84(4), 2734-2755.

[15] Dupont, C.H., Mazat J.P., Guerin, B., 1985. The role of adenine nucleotide translocation in the energization of the inner membrane of mitochondria isolated from rho + and rho degree strains of Saccharomyces cerevisiae. Biochem Biophys Res Commun, 132(3), 1116-23.

[16] Foulkes, E.C., 1955. Studies in cell permeability, the uptake of pyruvate by yeast. J. Gen. Physiol. $38(4), 425-430$.

[17] Garfinkel D., 1971. Simulation of the Krebs cycle and closely related metabolism in perfused rat liver. I Construction of a model. Comput. Biomed. Res. 4(1), 1-17.

[18] Garfinkel D., 1971. Simulation of the Krebs cycle and closely related metabolism in perfused rat liver. II Proprieties of the model. Comput. Biomed. Res. 4(1), 18-42.

[19] Greenhaff, P.L., Campbell-O'Sullivan, S. P., Constantin-Teodosiu, D., Poucher, S. M., Roberts, P. A., Timmons, J. A., 2002. An acetyl group deficit limits mitochondrial ATP production at the onset of exercise. Biochem. Soc. Trans. 30(2), 275-280.

[20] Guérin, B., 2004. Bioénérgetique. EDP Sciences, Grenoble, p.166.

[21] Gunter, T. E., Pfeiffer, D. R., 1990. Mechanisms by mitochondria transport calcium. Am. J. Physiol. 258, C755-C786.

[22] Guynn, R. W., Veech, R. L., 1973. The equilibrium constants of the adenosine triphosphate hydrolysis and the adenosine triphosphate-citrate lyase reactions. J. Biol. Chem. 248(20), 6966-6972.

[23] Hesselink, R.P., Van Kranenburg, G., Wagenmakers, A. J., Van der Vusse, G. J., Drost, M. R., 2005. Age related decline in muscle strength and power output in 1-4-a-glucosidase knockout mice. Muscle Nerve, 2005, 31(3), 374-381.

[24] Holian, A., Wilson, D.F., 1980. Relationship of transmembrane $\mathrm{pH}$ and electrical gradients with respiration and adenosine 5'-triphosphate synthesis in mitochondria. Biochem. 19(18), 4213-4221.

[25] Howarth, K.R., LeBlanc, P. J., Heigenhauser, G. J., Gibala, M. J., 2004. Effect of endurance training on muscle TCA cycle metabolism during exercise in humans. J. Appl. Physiol., 2004, 97(2), 579-584.

[26] Kondracheva, M. N., Doliba, N.M., 1989. Polarographic observation of substrate-level phosphorylation and its stimulation by acetylcholine. FEBS Letters 243, 153-155.

[27] Korzeniewski, B., 1991. An extended dynamic model of oxidative phosphorylation. Biochim. Biophys. Acta 1060(2), 210-223. 
[28] Korzeniewski, B., Malgat, M., Letellier, T., Mazat, J.P., 2001. Effect of 'binary mitochondrial heteroplasmy' on respiration and ATP synthesis: implications for mitochondrial diseases. Biochem J. 357, 835-42.

[29] Laplante, A., Compte, B., Des Rosiers, C. 1995. Assay of blood and tissue oxaloacetate and $\alpha$ ketogluterate by isotope dilution gas chromatography-mass spectrometry. Anal. Biochem. 224(2), 580-587.

[30] Lehninger Biochemistry, 1977. Worth Publisher, New York, 2nd edition.

[31] López-Soriano, J., Carbó, N., Almendro, V., Figueras, M., Ribas, V. Busquets, S., López-Soriano, F.j., 2004. Rat liver lipogenesis is modulated by interleukin-15. Int. J. Mol. Med. 13(6), 817-819.

[32] Magnus, G., Keizer, J., 1997. Minimal model of $\beta$-cell mitochondrial $C a^{2+}$-handling. Am. J. Physiol. Cell. Physiol. 273, C717-C733.

[33] Metzler, D.E., 1978. Biochemistry: The chemical reactions of living cells. Harcourt Academic Press, New York, 2nd edition.

[34] Ohanna M., Sobering A. K., Lapointe, T., Lorenzo L., Praud, C., Petroulakis, E., Sonenberg, N., Kelly, P. A., Sotiropoulos, A., Pende, M., 2005. Atrophy of S6K1(-/-) skeletal muscle cells reveals distinct mTOR effectors for cell cycle and size control. Nat. Cell Biol. 7(3), 286-294.

[35] Parlo, R. A., Coleman, P. S., 1984. Enhanced rate of citrate export from cholesterol-rich hepatoma mitochondria. J. Biol. Chem. 259,9997-10003.

[36] Pennisi, P. A., Kopchick, J. J., Thorgeirsson, S., LeRoith, D., Yakar, S., 2004. Role of growth hormone(GH)in liver regeneration. Endocrinology 145(10), 4748-4755.

[37] Putman, C. T., Matsos, M. P., Hultman, E., Jones, N. L., Heigenhauser, G. J., 1999. Pyruvate dehydrogenase activation in active muscle during and after maximal exercise in men. Am. J. Physiol. Endocrinol. Metab. 276(3), E483-E488.

[38] Ricny, J., Tucek, S., 1980. Relation between the content of acetyl-coenzyme A and acetylchloline in brane slices. Bioch. J. 188(3), 683-688.

[39] Rosenblad, C., Kirik, D., Björklund, A., 1999. Neurturin enhances the survival of intrastriatal fetal dopaminergic transplants. NeuroReport 10(8), 1783-1887.

[40] Rouzaire-Dubois, B., Dubois, J. M., 1998. K+ channel block-induced mammalian neuroblastoma cell swelling: a possible mechanism to influence profliferation. J. Physiol. 510, 93-102.

[41] Rustin, P., Bourgeron, T., Parfait, B., Chretien, D., Munnich, A., Rotig, A., 1997. Inborn errors of the Krebs cycle: a group of unusual mitochondrial diseases in human. Biochim. Biophys. Acta 1361, 185-197.

[42] Saks, V. A., Kaambre, T., Sikk, P., Eimre, M. Orlova, E., Paju, K., Piirsoo, A. Appaix, F. Kay, L., Regitz-Zagrosek, V., Fleck, E., Seppet, E., 2001. Intracellular energetic units in red muscle cells.Biochem. J. 356, 643-657.

[43] Schneider, S.W., Yano, Y., Sumpio, B. E., Jena, B. P., Geibel, J. P., Gekle, M., Oberleithner, H., 1997. Rapid aldosterone-induced cell volume increase of endothelial cells measured by the atomic force microscope. Cell Biol. Int. 21(11), 759-768.

[44] Segel, I., 1975. Enzyme kinetics : behavior and analysis of rapid equilibrium and steady state enzyme systems. Wiley, New York, 1975.

[45] Sies, H., 1982. Metabolic compartimentation. Academic Press, London, 123-146 and 235-257.

[46] Smoller, J., 1994, Shock waves and reaction-diffusion equations, Springer-Verlag, 2nd edition.

[47] Stamenovic, D., Mijailovich, S. M., Tolic-Norrelykke, I. M., Chen, J., Wang, N. 2002. Cell prestress. II. Contribution of microtubules. Am. J. Physiol. Cell Physiol. 282(3), C617-C624.

[48] Berg, J.M., Tymoczko, J. and Stryer L., 2006. Biochemistry, W.H. Freeman \& Co. 
[49] Viant, M. R., Rosenblum, E. S., Tieerdema, R. S., 2003. NMR-based metabolomics : a powerful approach for characterizing the effects of environmental stressors on organism health.Environ. Sci. Technol. 37(21), 4982-4989.

[50] Wilson, D. F., Owen, C. S., Holian, A., 1977. Control of mitochondrial respiration: a quantitative evaluation of the roles of cytochrome c and oxygen. Arch. Biochem. Biophys. 182(2), 749-762.

[51] Wojtczak, L., Zolkiewska, A., Duszynski, J., 1986. Energy-storage capacity of the mitochondrial proton-motive force. Biochim. Biophys. Acta, 851(2), 313-321.

[52] Wright BE, Butler MH, Albe KR., 1992. Systems analysis of the tricarboxylic acid cycle in Dictyostelium discoideum. I. The basis for model construction. J. Biol. Chem. 267(5), 3101-3105.

[53] Wu, F., Yang, F., Vinnakota, K. C., Beard, D. A., 2007. Computer modelling of mitochondrial tricarboxylic cycle, oxidative phosphorylation, metabolite transport, and electrophysiology. J. Biol. Chem. 282(34), 24525-24537.

[54] Yeh, L.A., Song, C. S., Kim, K. H., 1981. Coenzyme A activation of acetyl-CoA carboxylase. J. Biol. Chem., 1981, 256(5), 2289-2296.

[55] Zeidan, A., Nordström, I., Dreja, K., Malmqvist, U., Hellstrand, P., 2000. Stretch-dependent modulation of contractility and growth in smooth muscle of rat portal vein. Circ. Res. 87(3), 228-234.

[56] Zupke, C., Sinskey, A. J., Stephanopoulos, G., 1995. Intracellular flux analysis applied to the effect of dissolved oxygen on hybridomas. Appl. Microbiol. Biotechnol. 44(1-2), 27-36. 